\title{
ÁREA IRRIGADA POR PIVÔ CENTRAL NO MUNICÍPIO DE PINHEIROS, ES
}

\author{
Rebyson Bissaco Guidinelle ${ }^{1}$ \\ Alex Justino Zacarias ${ }^{2}$ \\ Israel Martins Pereira ${ }^{3}$ \\ Maxwel Rodrigues Nascimento ${ }^{4}$ \\ Julio Cesar Gradice Saluci ${ }^{5}$ \\ Mário Euclides Pechara da Costa Jaeggi ${ }^{6}$ \\ Rogério Rangel Rodrigues ${ }^{7}$ \\ Samuel Ferreira da Silva ${ }^{8}$ \\ Jéferson Luiz Ferrari ${ }^{9}$
}

Resumo: Este trabalho teve como objetivo realizar o levantamento da área irrigada com pivô central no município de Pinheiros, Espírito Santo, Brasil. O levantamento da área irrigada por pivô central foi realizado mediante o uso do programa Google Earth ${ }^{T M}$ Pro. Foi feito a sobreposição do arquivo vetorial do município de Pinheiros sobre a imagem de satélite disponível no programa computacional. Em seguida realizou-se a fotointerpretação de todas as áreas irrigadas com pivô central. A escala utilizada foi de 1:1.000. Foram levantados o raio $(\mathrm{m})$, o perímetro $(\mathrm{m})$ e a área de cada alvo fotointerpretado. Chegando à uma conclusão que o município de Pinhiros possui 116 áreas irrigadas por pivô central, totalizando uma área de 5624,40 ha.

Palavras-chave: Geoprocessamento; Google Earth ${ }^{T M}$ Pro; Fotointerpretação; Mapa de uso da terra.

\footnotetext{
${ }^{1}$ Graduando em Tecnologia em Cafeicultura/Instituto Federal do Espírito Santo, Campus de Alegre, IFES - ES, Brasil. E- mail: rebysonguidinelle@gmai.com.

2 Graduando em Tecnologia em Cafeicultura/Instituto Federal do Espírito Santo, Campus de Alegre, IFES - ES, Brasil. E-mail: alexjustino12@gmail.com.

${ }^{3}$ Graduando em Tecnologia em Cafeicultura/Instituto Federal do Espírito Santo, Campus de Alegre, IFES - ES, Brasil. E- mail: israelmartins80@gmail.com.

4 Pós- Graduando em Produção Vegetal/Universidade Estadual Norte Fluminense - RJ, Brasil. E-mail: maxwel.m88@gmail.com.

${ }^{5}$ Graduando em Tecnologia em Cafeicultura/Instituto Federal do Espírito Santo, Campus de Alegre, IFES - ES, Brasil. E- mail: juliosaluci@gmail.com.

6 Pós- Graduando em Produção Vegetal/Universidade Estadual Norte Fluminense - RJ, Brasil. E-mail: mariopechara@hotmail.com.

7 Departamento de Engenharia/Universidade Federal de Lavras, Lavras - MG, Brasil. E-mail: rogeriorr7@hotmail.com.

8 Pós- Graduação em Produção Vegetal, Universidade Federal do Espírito Santo, Campus de Alegre, UFES - ES, Brasil. E-mail: samuelfd.silva@yahoo.com.br.

9 D.Sc. Professor/Instituto Federal do Espírito Santo, Campus de Alegre, IFES - ES, Brasil. E-mail: ferrarijluiz@gmail.com.
} 